\title{
Spatial Risk Measures: Local Specification and Boundary Risk
}

\author{
Hans Föllmer and Claudia Klüppelberg
}

\begin{abstract}
We study a mathematical consistency problem motivated by the interplay between local and global risk assessment in a large financial network. In analogy to the theory of Gibbs measures in Statistical Mechanics, we focus on the structure of global convex risk measures which are consistent with a given family of local conditional risk measures. Going beyond the locally law-invariant (and hence entropic) case studied in [11], we show that a global risk measure can be characterized by its behavior on a suitable boundary field. In particular, a global risk measure may not be uniquely determined by its local specification, and this can be seen as a source of "systemic risk", in analogy to the appearance of phase transitions in the theory of Gibbs measures. The proof combines the spatial version [10] of Dynkin's method for constructing the entrance boundary of a Markov process with the non-linear extension [14] of backwards martingale convergence.
\end{abstract}

\section{Introduction}

In a large network of financial institutions, the risk at a given node of the network is usually assessed in terms of some monetary risk measure that involves the marginal distribution at that node. But such an approach neglects the interactive effects that are not captured by the family of marginal distributions. This suggests to take a conditional approach, where the risk measure applied at a given node takes into account the situation at the other nodes of the network; see, for example, [1]. The question is whether these conditional risk measures can be aggregated in a consistent

Hans Föllmer

Institut für Mathematik Humboldt Universität zu Berlin, Unter den Linden 6, Germany D-10099

Berlin, e-mail: foellmer@math.hu-berlin.de

Claudia Klüppelberg

Center for Mathematical Sciences, Technische Universität München, 85748 Garching, Boltzmannstrasse 3, Germany, e-mail: cklu@tum.de 
manner to a global risk measure, and whether the global risk measure is uniquely determined by the local specification.

With this motivation in mind, we are going to focus on some of the purely mathematical problems which arise in such a spatial setting, and which can be viewed as non-linear analogues to some classical problems in the theory of Gibbs measures. In Dobrushin's probabilistic approach to the analysis of phase transitions in Statistical Mechanics, Gibbs measures are specified by a consistent family of local conditional probability distributions; cf. [6] or [18]. In an infinite spatial network, the global Gibbs measure may not be uniquely determined by the local specification. Nonuniqueness is interpreted as a phase transition, and in that case Gibbs measures can be described as mixtures of phases, defined as extreme points in the convex set of all Gibbs measures.

In analogy to Dobrushin's approach, we start with a given family $\left(\rho_{V}\right)_{V \in \mathscr{V}}$ of local conditional risk measures indexed by the class $\mathscr{V}$ of finite subsets of some infinite set of nodes. These conditional risk measures are convex, and they are assumed to be consistent in the usual sense, that is, $\rho_{W}\left(-\rho_{V}\right)=\rho_{W}$ if $V \subseteq W$. Our aim is to clarify the structure of the set $\mathscr{R}$ of global convex risk measures which are consistent with this local specification.

To this end, we assume that the local conditional risk measures $\rho_{V}$ are absolutely continuous with respect to the local conditional probability distributions $\pi_{V}$ in the local specification of a Gibbs measure. Under the stronger assumption of local law invariance, the conditional risk of a financial position $X$ would only depend on the distribution of $X$ under the conditional probability measure $\pi_{V}$. As shown in [11], the local risk measures must then be entropic, and the representation of global risk measures can be described in a rather explicit manner.

In this paper we go beyond the special case of local law invariance. But then the main difficulty consists in extending the local specification $\left(\rho_{V}\right)_{V \in \mathscr{V}}$ to a sufficiently regular conditional risk measure with respect to the tail field. We solve this problem by combining two methods. On the one hand, we use the supermartingale properties implied by local consistency, and in particular the non-linear extension of backwards martingale convergence developed in [14]. On the other hand, we use Dynkin's method $[8,9]$ of constructing the entrance boundary of a Markov process, adapted to our spatial setting as in [10]. In this way, the set of phases can be described as a spatial "boundary", defined by a sub- $\sigma$-field $\hat{\mathscr{F}}$ of the tail field. As our main result, we show that a sufficiently regular global risk measure $\rho$ in $\mathscr{R}$ is uniquely determined by its behavior on the boundary field $\hat{\mathscr{F}}$. In particular, we show that we have non-uniqueness of the global risk measure if the underlying probabilistic structure admits a phase transition. From a financial point of view, this can be viewed as one mathematical aspect of the much broader issue of "systemic risk".

The paper is organized as follows. In Section 2 we recall some basic facts from the theory of convex risk measures, and in particular the notion of a convex risk kernel introduced in [11]. In Section 3 we describe our spatial setting and the local specification of convex risk measures in terms of local risk kernels. The extension of this local specification to a sufficiently regular convex risk kernel with respect to the tail field is done in two steps. In Section 4 we use a straightforward definition of 
a limiting kernel $\rho_{\infty}$ and show that it has good properties with respect to any given Gibbs measure $P$. But this kernel does not behave well enough simultaneously for all such Gibbs measures. To overcome this difficulty, we introduce an additional regularization that involves Dynkin's boundary construction. This second step is carried out in Section 5, and the resulting risk kernel $\hat{\rho}_{\infty}$ is shown to be the key to the structure of global risk measures.

\section{Preliminaries on convex risk kernels}

In this section we recall some basic definitions and facts from the theory of convex risk measures initiated in [2], [16], and [17], and also the notion of a convex risk kernel introduced in [11]. For more details see, for example, [12] and [15].

Let $(\Omega, \mathscr{F})$ be a measurable space, and denote by $M:=M_{b}(\Omega, \mathscr{F})$ the space of all bounded measurable functions on $(\Omega, \mathscr{F})$. A real-valued functional $\rho$ on $M$ is called a monetary risk measure if it is monotone, i.e., $\rho(X) \geq \rho(Y)$ whenever $X \leq Y$, cash-invariant, i.e., $\rho(X+m)=\rho(X)-m$ for constants $m$, and normalized, i.e., $\rho(0)=0$. If a monetary risk measure $\rho$ is also convex on $M$, then $\rho$ will be called a convex risk measure. A convex risk measure is called coherent if it is also positively homogeneous, that is, $\rho(\lambda X)=\lambda \rho(X)$ for any positive constant $\lambda$. We denote by $\mathscr{A}:=\{X \in M \mid \rho(X) \leq 0\}$ the acceptance set of $\rho$; in the convex case the acceptance set is convex, in the coherent case a convex cone.

Typically, a convex risk measure has a dual representation

$$
\rho(X)=\sup _{Q \in \mathscr{Q}}\left(E_{Q}[-X]-\alpha(Q)\right),
$$

in terms of some set $\mathscr{Q}$ of probability measures on $(\Omega, \mathscr{F})$ and some penalty function $\alpha: \mathscr{Q} \rightarrow[0, \infty]$. In this case, the representation also holds if we choose

$$
\alpha(Q)=\sup _{X \in \mathscr{A}} E_{Q}[-X]
$$

and this is the minimal penalty function such that (1) holds.

A necessary condition for (1) is the Fatou property of $\rho$, that is,

$$
\lim _{k \rightarrow \infty} X_{k}=X \quad \text { pointwise } \Longrightarrow \rho(X) \leq \liminf _{k \rightarrow \infty} \rho\left(X_{k}\right)
$$

for any uniformly bounded sequence $\left(X_{k}\right)_{k=1,2, \ldots}$ in $M$; cf. [15], Lemma 4.21. We say that $\rho$ has the Lebesgue property if (3) is replaced by the stronger condition

$$
\lim _{k \rightarrow \infty} X_{k}=X \quad \text { pointwise } \Longrightarrow \rho(X)=\lim _{k \rightarrow \infty} \rho\left(X_{k}\right)
$$


This condition is sufficient for the dual representation of $\rho$, and it implies that the supremum in (1) is actually attained; cf. [15] Th. 4.22 and Exercise 4.22.

Now let $P$ be a probability measure on $(\Omega, \mathscr{F})$.

Definition 1. If $\rho$ is a monetary risk measure on $M$ such that $\rho(X)=\rho(Y)$ whenever $X=Y P$-almost surely, then we say that $\rho$ is absolutely continuous with respect to $P$, and we write

$$
\rho \ll P
$$

In this case, $\rho$ can also be considered as a monetary risk measure on the Banach space $L^{\infty}(\Omega, \mathscr{F}, P)$. Such a risk measure is called law-invariant with respect to $P$ if $\rho(X)=\rho(Y)$ whenever $X$ and $Y$ have the same distribution under $P$.

If $\rho \ll P$ then the Fatou property is both necessary and sufficient for the dual representation (1) of $\rho$, regarded as a convex risk measure on $L^{\infty}(\Omega, \mathscr{F}, P)$. In this case we have $Q \ll P$ for any $Q$ such that $\alpha(Q)<\infty$, and so we can restrict $\mathscr{Q}$ to the class of probability measures which are absolutely continuous with respect to $P$; see Theorem 4.33 in [15]. If $\rho$ satisfies the stronger Lebesgue property, then the supremum in (1) is actually attained by some $Q \ll P$ depending on $X$; see Corollary 4.35 in [15], and also [5] for a converse result.

Example 1. Let $P$ be a probability measure $P$ on $(\Omega, \mathscr{F})$, and consider the entropic risk measure $e_{\beta}$ with parameter $\beta \in[0, \infty)$, defined by

$$
e_{\beta}(X)=\frac{1}{\beta} \log E_{P}\left[e^{-\beta X}\right]
$$

for $\beta=0$, this will be interpreted as the limiting linear case

$$
e_{0}(\beta):=\lim _{\beta \downarrow 0} e_{\beta}(X)=E_{P}[-X]
$$

An entropic risk measure is clearly convex and law-invariant. It has the Lebesgue property, and the minimal penalty function in its dual representation (1) is given by

$$
\alpha(Q)=\frac{1}{\beta} H(Q \mid P),
$$

where $H(Q \mid P)$ denotes the relative entropy of $Q$ with respect to $P$; for $\beta=0$ the penalty function is to be read as 0 if $Q=P$ and as $+\infty$ if not.

Let $\mathscr{F}_{0} \subseteq \mathscr{F}$ be a sub- $\sigma$-field of $\mathscr{F}$, and denote by $M_{0}$ the space of bounded measurable functions on $\left(\Omega, \mathscr{F}_{0}\right)$. Let us first recall the definition of a stochastic kernel $\pi(\omega, d \eta)$ from $\left(\Omega, \mathscr{F}_{0}\right)$ to $(\Omega, \mathscr{F})$ : For any $\omega \in \Omega, \pi(\omega, \cdot)$ is a probability measure on $(\Omega, \mathscr{F})$, and for any $A \in \mathscr{F}$, the function $\pi(\cdot, A)$ on $\Omega$ is $\mathscr{F}_{0}$-measurable. For a probability measure $P$ on $\left(\Omega, \mathscr{F}_{0}\right)$ we denote by $P \pi$ the probability measure on $(\Omega, \mathscr{F})$ defined by $P \pi[A]=\int \pi(\omega, A) P(d \omega)$. The stochastic kernel will be called regular if $\pi(\omega, \cdot)=\delta_{\omega}$ on $\mathscr{F}_{0}$. For two such kernels $\pi_{i} \quad(i=0,1)$, their composition $\pi_{0} \pi_{1}$ is defined as the stochastic kernel given by $\pi_{0} \pi_{1}(\omega, A)=\int \pi_{1}(\eta, A) \pi_{0}(\omega, d \eta)$. 
Let us now extend the classical definition of a stochastic kernel in the following manner.

Definition 2. A monetary risk kernel from $\left(\Omega, \mathscr{F}_{0}\right)$ to $(\Omega, \mathscr{F})$ is a real-valued function $\rho_{0}$ on $\Omega \times M$ such that

i) for each $\omega \in \Omega$, the functional $\rho_{0}(\omega, \cdot)$ is a monetary risk measure on $M$;

ii) for each $X \in M$, the function $\rho_{0}(\cdot, X)$ belongs to $M_{0}$.

Such a monetary risk kernel $\rho_{0}$ will be called convex if all risk measures $\rho_{0}(\omega, \cdot)$ are convex. It will be called regular if

$$
\rho_{0}\left(\omega, f\left(X_{0}, X\right)\right)=\rho_{0}\left(\omega, f\left(X_{0}(\omega), X\right)\right)
$$

for $\omega \in \Omega, X_{0} \in M_{0}, X \in M$, and for any bounded measurable function $f$ on $R^{2}$.We will say that the risk kernel $\rho_{0}$ has the Fatou property, or the Lebesgue property, if condition (3) or condition (4) holds for each risk measure $\rho_{0}(\omega, \cdot)$.

Note that regularity of a monetary risk kernel $\rho_{0}$ from $\left(\Omega, \mathscr{F}_{0}\right)$ to $(\Omega, \mathscr{F})$ implies the following local property:

$$
\rho_{0}\left(\omega, I_{A_{0}} X+I_{A_{0}^{c}} Y\right)=I_{A_{0}}(\omega) \rho_{0}(\omega, X)+I_{A_{0}^{c}}(\omega) \rho_{0}(\omega, Y)
$$

for $\omega \in \Omega, X, Y \in M$, and any $A_{0} \in \mathscr{F}_{0}$.

The composition $\rho_{0}\left(-\rho_{1}\right)$ of two monetary risk kernels $\rho_{0}$ and $\rho_{1}$ is defined as the monetary risk kernel given by

$$
\left(\rho_{0}\left(-\rho_{1}\right)\right)(\omega, X):=\rho_{0}\left(\omega,-\rho_{1}(\cdot, X)\right) .
$$

If $\rho_{0}$ and $\rho_{1}$ are both convex, then their composition $\rho_{0}\left(-\rho_{1}\right)$ is again convex.

If $\rho_{0}$ is a regular convex risk kernel from $\left(\Omega, \mathscr{F}_{0}\right)$ to $(\Omega, \mathscr{F})$ such that the risk measures $\rho_{0}(\omega, \cdot)$ satisfy the condition

$$
\rho_{0}(\omega, \cdot) \ll P \quad P \text {-a.s. }
$$

then it is easy to check that $\rho_{0}$ can be regarded as a conditional convex risk measure in the usual sense, as specified by the following definition.

Definition 3. A map $\rho_{0}$ from $L^{\infty}(\Omega, \mathscr{F}, P)$ to $L^{\infty}(\Omega, \mathscr{F} 0, P)$ is called a conditional monetary risk measure with respect to $\mathscr{F}_{0}$ and $P$, if it satisfies the following three properties for any $X, Y \in L^{\infty}(\Omega, \mathscr{F}, P)$ :

i) Monotonicity: $\rho_{0}(X) \geq \rho_{0}(Y) P$-a.s. whenever $X \leq Y P$-a.s.;

ii) Conditional cash invariance: $\rho_{0}(X+m)=\rho_{0}(X)-m P$-a.s. for all $m \in L^{\infty}\left(\Omega, \mathscr{F}_{0}, P\right)$;

iii) Normalization: $\rho_{0}(0)=0$-a.s..

Such a conditional risk measure $\rho_{0}$ is called convex if 


$$
\rho_{0}(\lambda X+(1-\lambda) Y) \leq \lambda \rho_{0}(X)+(1-\lambda) \rho_{0}(Y) \quad P-\text { a.s. }
$$

for any $\mathscr{F}_{0}$-measurable function $\lambda$ such that $0 \leq \lambda \leq 1 \quad P$-a.s.. It is said to have the Fatou property if

$$
\lim _{k \rightarrow \infty} X_{k}=X P \text {-a.s. } \Longrightarrow \rho(X) \leq \liminf _{k \rightarrow \infty} \rho\left(X_{k}\right) P-\text { a.s. }
$$

for any uniformly bounded sequence $\left(X_{k}\right)_{k=1,2, .}$ in $L^{\infty}(\Omega, \mathscr{F}, P)$; the Lebesgue property is defined in the same manner.

Note that the Fatou or the Lebesgue property of the risk measures $\rho_{0}(\omega, \cdot)$ in (9) implies the corresponding property of $\rho_{0}$, regarded as a conditional risk measure with respect to $P$.

If a convex conditional risk measure $\rho_{0}$ with respect to $\mathscr{F}_{0}$ and $P$ has the Fatou property, then it admits a conditional version of the dual representation (1). Denoting by

$$
\mathscr{A}_{0}:=\left\{X \in L^{\infty}(\Omega, \mathscr{F}, P) \mid \rho_{0}(X) \leq 0 P-\text { a.s. }\right\}
$$

the acceptance set of $\rho_{0}$, the dual representation takes the form

$$
\rho_{0}(X)=\operatorname{ess} \sup \left(E_{Q}\left[-X \mid \mathscr{F}_{0}\right]-\alpha_{0}(Q)\right),
$$

where the essential supremum is taken with respect to $P$ and over all probability measures $Q \ll P$ such that $Q \approx P$ on the $\sigma$-field $\mathscr{F}_{0}$, and where the minimal penalty function is given by

$$
\alpha_{0}(Q)=\operatorname{ess} \sup _{X \in \mathscr{A}_{0}} E_{Q}\left[-X \mid \mathscr{F}_{0}\right],
$$

see [7] or Theorem 11.2 in [16]. For a general $Q \ll P$, (11) is defined as an essential supremum under $Q$. But if $Q$ satisfies the additional condition $Q \approx P$ on $\mathscr{F}_{0}$ as in (10), then it can as well be read as an essential supremum under $P$.

\section{Local specification of spatial risk measures}

Let $I$ be a countable set of sites, and let $S$ be some Polish state space with Borel $\sigma-$ field $\mathscr{S}$. We assume that each site $i \in I$ can be in some state $s \in S$, and we denote by $\Omega=S^{I}$ the set of possible configurations $\omega: I \rightarrow S$. For any subset $J \subseteq I$, we denote by $\omega_{J}$ the restriction of $\omega$ to $J$, by $\mathscr{F}_{J}$ the $\sigma$-field on $\Omega$ generated by the projection maps $\omega \rightarrow \omega(i)$ for any $i \in J$, and we write $\mathscr{F}=\mathscr{F}_{I}$. A probability measure $P$ on $(\Omega, \mathscr{F})$ is also called a random field.

Let $\mathscr{V}$ denote the class of non-empty finite subsets $V \subseteq I$. For a given set $V \in \mathscr{V}$, the $\sigma$-field $\mathscr{F}_{V}$ describes what is observable on $V$, while $\mathscr{F}_{V c}$ describes the situation on $V^{c}:=I \backslash V$, also called the environment of $V$. 
Definition 4. A collection $\left(\rho_{V}\right)_{V \in \mathscr{V}}$ of regular convex risk kernels $\rho_{V}$ from $\left(\Omega, \mathscr{F}_{V^{c}}\right)$ to $(\Omega, \mathscr{F})$ is called a local specification of a convex risk measure if it satisfies the consistency condition

$$
\rho_{W}\left(-\rho_{V}\right)=\rho_{W}
$$

for any $V, W \in \mathscr{V}$ such that $V \subseteq W$, and if each risk kernel $\rho_{V}$ is regular in the sense of (7) and has the Fatou property.

From now on we fix a local specification $\left(\rho_{V}\right)_{V \in \mathscr{V}}$ of a convex risk measure.

Definition 5. Let $\mathscr{R}$ denote the set of all convex risk measures $\rho$ on $M$ which are consistent with the local specification $\left(\rho_{V}\right)_{V \in \mathscr{V}}$, that is,

$$
\rho\left(-\rho_{V}\right)=\rho \quad \text { for any } V \in \mathscr{V} .
$$

Our aim is to clarify the structure of the global risk measures in $\mathscr{R}$. At the general level of Definition 4 there is not much to be said. The situation becomes more transparent if we introduce an underlying probabilistic structure, described by the local specification of a random field; cf. [6] and [18].

Definition 6. A collection $\left(\pi_{V}\right)_{V \in \mathscr{V}}$ of regular stochastic kernels $\pi_{V}$ from $\left(\Omega, \mathscr{F}_{V^{c}}\right)$ to $(\Omega, \mathscr{F})$ is called a local specification of a random field if it satisfies the consistency condition

$$
\pi_{W} \pi_{V}=\pi_{W}
$$

for any $V, W \in \mathscr{V}$ such that $V \subseteq W$.

Definition 7. We denote by $\mathscr{P}$ the convex set of all random fields $P$ which are consistent with this local specification in the sense that

$$
P \pi_{V}=P \quad \text { for any } V \in \mathscr{V} \text {. }
$$

A random field $P \in \mathscr{P}$ is also called a Gibbs measure. The case $|\mathscr{P}|>1$, where the global random field is not uniquely determined by the local specification $\left(\pi_{V}\right)_{V \in \mathscr{V}}$, is often referred to as a phase transition.

For any $V \in \mathscr{V}$, the stochastic kernel $\pi_{V}$ serves as a conditional probability distribution with respect to $\mathscr{F}_{V^{c}}$ which is common to all probability measures $P \in \mathscr{P}$, and so we can write

$$
E_{P}\left[f \mid \mathscr{F}_{V^{c}}\right](\omega)=\int f(\eta) \pi_{V}(\omega, d \eta)
$$

for any $P \in \mathscr{P}$ and any measurable function $f \geq 0$ on $(\Omega, \mathscr{F})$.

Let us now fix a local specification $\left(\pi_{V}\right)_{V \in \mathscr{V}}$ of a random field such that

$$
\mathscr{P} \neq \emptyset \text {. }
$$


We connect our local specification $\left(\rho_{V}\right)_{V \in \mathscr{V}}$ of a convex risk measure with the local specification $\left(\pi_{V}\right)_{V \in \mathscr{V}}$ by the following assumption:

Assumption 1. For any $\omega \in \Omega$ and any $V \in \mathscr{V}$, the convex risk measure $\rho_{V}(\omega, \cdot)$ has the following two properties:

i) $\rho_{V}(\omega, \cdot) \ll \pi_{V}(\omega, \cdot)$

ii) If $X$ is acceptable for $\rho_{V}(\omega, \cdot)$ then the expected loss under the measure $\pi_{V}(\omega, \cdot)$ is uniformly bounded from above, i.e., there is a constant $c \geq 0$ such that

$$
\rho_{V}(\omega, X) \leq 0 \Longrightarrow \int(-X)(\eta) \pi_{V}(\omega, d \eta) \leq c .
$$

Remark 1. The local specification $\left(\rho_{V}\right)_{V \in \mathscr{V}}$ is called law-invariant if Assumption 1(i) is replaced by the much stronger assumption that each convex risk measure $\rho_{V}(\omega, \cdot)$ is law-invariant with respect to the probability measure $\pi_{V}(\omega, \cdot)$. This implies

$$
\rho_{V}(\omega, X) \geq \int(-X)(\eta) \pi_{V}(\omega, d \eta)
$$

for any $X \in M$, and so condition (18) is satisfied with $c=0$; see Corollary 4.65 in [16]. Actually much more is true: Under mild regularity conditions, local law invariance together with consistency of the family $\left(\rho_{V}\right)_{V \in \mathscr{V}}$ implies that the risk measures $\rho_{V}(\omega, \cdot)$ must be entropic; see [10] and also [19]. More precisely, the risk kernel $\rho_{V}$ takes the form

$$
\rho_{V}(\omega, X)=\frac{1}{\beta_{\infty}(\omega)} \log \int e^{-\beta_{\infty}(\omega) X(\eta)} \pi_{V}(\omega, d \eta)
$$

with $\beta_{\infty}(\omega) \in[0, \infty)$, as in Example 1. Due to consistency, the parameter $\beta_{\infty}(\omega)$ does not depend on $V$, and this implies that the function $\beta_{\infty}(\cdot)$ is measurable with respect to the tail field $\mathscr{F}_{\infty}$ introduced in Section 4 below; see [10] for more details.

Lemma 1. For any $P \in \mathscr{P}$, the risk kernel $\rho_{V}$ can be regarded as a conditional risk measure

$$
\rho_{V}: L^{\infty}(\Omega, \mathscr{F}, P) \rightarrow L^{\infty}\left(\Omega, \mathscr{F}_{V^{c}}, P\right),
$$

and this conditional risk measure has the Fatou property with respect to $P$.

Proof. Take $X$ and $Y$ in $M$ such that $X=Y P$-a.s.. We have to show that $\rho_{V}(\cdot, X)=$ $\rho_{V}(\cdot, Y) P$-a.s.. Indeed, the consistency condition $P=P \pi_{V}$ implies $\pi_{V}(\cdot, X)=$ $\pi_{V}(\cdot, Y) P$-a.s., hence $\rho_{V}(\cdot, X)=\rho_{V}(\cdot, Y) P$-a.s. due to part i) of our Assumption 1. The Fatou property of the conditional risk measure with respect to $P$ follows from the Fatou property of the risk kernel $\rho_{V}$.

We now take a closer look at our consistency condition (12). For a given probability measure $P \in \mathscr{P}$, this can be read as a consistency condition for two conditional risk measures with respect to $P$, as shown by Lemma 1 . As such, it can be characterized at the level of the corresponding acceptance sets and also at the level of penalty 
functions; see, for example, [4] and [13]. For our purposes, however, we will need an alternative characterization in terms of the following supermartingale property; see [13] and Theorem 2 in [3].

Proposition 1. For any $P \in \mathscr{P}$ and any $V, W \in \mathscr{V}$ such that $V \subseteq W$, the consistency condition $\rho_{W}\left(-\rho_{V}\right)=\rho_{W}$ yields the supermartingale inequality

$$
\rho_{W}(X)+\alpha_{W}(Q) \geq E_{Q}\left[\rho_{V}(X)+\alpha_{V}(Q) \mid \mathscr{F}_{W^{c}}\right] \quad P \text { - a.s. }
$$

for any $X \in L^{\infty}(\Omega, \mathscr{F}, P)$ and any probability measure $Q \ll P$.

\section{Passing to the tail field}

Our aim is to clarify the structure of the class $\mathscr{R}$ of global convex risk measures which are consistent with our local specification $\left(\rho_{V}\right)_{V \in \mathscr{V}}$, in analogy to the classical analysis of the class $\mathscr{P}$ of global random fields which are consistent with the local specification $\left(\pi_{V}\right)_{V \in \mathscr{Y}}$.

This problem is trivial if $I$ is finite: In this case we have $I \in \mathscr{V}$ and $\mathscr{F}_{I^{c}}=\{\emptyset, \Omega\}$, and so $\rho_{I}(\omega, \cdot)$ does not depend on $\omega$. Thus there is exactly one risk measure $\rho \in \mathscr{R}$, namely $\rho=\rho_{I}$.

From now we assume $|I|=\infty$, and so $(\Omega, \mathscr{F})$ is an infinite product space. Here we will proceed in two steps. In this section we are going to extend the local specification $\left(\rho_{V}\right)_{V \in \mathscr{V}}$ in a consistent manner to a risk kernel $\rho_{\infty}$ with respect to the tail field

$$
\mathscr{F}_{\infty}:=\bigcap_{V \in \mathscr{V}} \mathscr{F}_{V^{c}}
$$

and we shall describe the properties of $\rho_{\infty}$ as a conditional risk measure with respect to any given measure $P \in \mathscr{P}$. The second step will be done in the next section. It involves a regularization of the initial kernel $\rho_{\infty}$, and this will be the key to the structure of global risk measures.

Let us fix a sequence $\left(V_{n}\right) \subseteq \mathscr{V}$ increasing to $I$, and let us use the notation

$$
\rho_{n}:=\rho_{V_{n}}, \quad n=1,2, \ldots
$$

for the corresponding sequence of convex risk kernels. Now consider the risk kernel $\rho_{\infty}$ defined by

$$
\rho_{\infty}(\omega, X):=\limsup _{n \rightarrow \infty} \rho_{n}(\omega, X)
$$

for any $X \in M$ and any $\omega \in \Omega$. We denote by

$$
M_{\infty}:=M_{b}\left(\Omega, \mathscr{F}_{\infty}\right)
$$


the space of all bounded measurable functions on $\left(\Omega, \mathscr{F}_{\infty}\right)$. For any $X \in M$, the function $\rho_{\infty}(\cdot, X)$ belongs to $M_{\infty}$, since it is bounded by $\|X\|$ and clearly measurable with respect to the tail field $\mathscr{F}_{\infty}$.

Lemma 2. The functional $\rho_{\infty}: M \rightarrow M_{\infty}$ defined by (21) is a regular convex risk kernel from $\left(\Omega, \mathscr{F}_{\infty}\right)$ to $(\Omega, \mathscr{F})$, and it satisfies the consistency condition

$$
\rho_{\infty}\left(-\rho_{V}\right)=\rho_{\infty}
$$

for any $V \in \mathscr{V}$.

Proof. For any $\omega \in \Omega$, the functional $\rho_{\infty}(\omega, \cdot)$ on $M$ inherits from the sequence $\left(\rho_{n}\right)$ the properties of a convex risk measure and also the regularity property (7). Moreover, we have

$$
\rho_{\infty}\left(-\rho_{V}(X)\right)=\limsup _{n \rightarrow \infty} \rho_{n}\left(-\rho_{V}(X)\right)=\limsup _{n \rightarrow \infty} \rho_{n}(X)=\rho_{\infty}(X)
$$

for any $V \in \mathscr{V}$, since $\rho_{n}\left(-\rho_{V}(X)\right)=\rho_{n}(X)$ as soon as $V \subset V_{n}$, due to the consistency condition (12).

For the rest of this section we fix a probability measure $P \in \mathscr{P}$. We are going to show that the limit superior in (21) is $P$-almost surely a limit, and that $\rho_{\infty}$ has good properties as a conditional risk measure with respect to $P$.

Lemma 1 shows that each risk kernel $\rho_{n}$ can be regarded as a conditional risk measure under $P$ with respect to $\mathscr{F}_{V_{n}^{c}}$, and that it has the Fatou property with respect to $P$. We denote by

$$
\mathscr{A}_{n}(P):=\left\{X \in L^{\infty}(\Omega, \mathscr{F}, P) \mid \rho_{n}(X) \leq 0 \quad P-\text { a.s. }\right\}
$$

its acceptance set and by

$$
\alpha_{n}(Q)=\operatorname{ess} \sup _{X \in \mathscr{A}_{n}(P)} E_{Q}\left[-X \mid \mathscr{F}_{V_{n}^{c}}\right] .
$$

its penalty function. It follows that $\rho_{n}$ admits the dual representation

$$
\rho_{n}(X)=\operatorname{ess} \sup \left(E_{Q}\left[-X \mid \mathscr{F}_{V_{n}^{c}}\right]-\alpha_{n}(Q)\right),
$$

where the essential supremum is taken over all $Q \ll P$ such that $Q \approx P$ on $\mathscr{F}_{n} V_{n}^{c}$. Let us also introduce the set

$$
\mathscr{Q}(P):=\left\{Q \in \mathscr{M}_{1}(P) \mid Q=P \text { on } \mathscr{F}_{\infty}, \sup _{\mathrm{n}} \mathrm{E}_{\mathrm{Q}}\left[\alpha_{\mathrm{n}}(\mathrm{Q})\right]<\infty\right\} .
$$

As we shall see in the proof of the following Proposition, we have $P \in \mathscr{Q}(P)$, hence $\mathscr{Q}(P) \neq \emptyset$.

Lemma 3. For any $Q \in \mathscr{Q}(P)$, the limit

$$
\alpha_{\infty}(Q)=\lim _{n \rightarrow \infty} \alpha_{n}(Q)
$$


exists $P$-a.s. and satisfies

$$
E_{P}\left[\alpha_{\infty}(Q)\right]<\infty .
$$

Proof. Take $Q \in \mathscr{Q}(P)$. Applying Proposition 1 for $X=0$, we see that the consistency condition $\rho_{n+1}=\rho_{n+1}\left(-\rho_{n}\right)$ implies the backwards supermartingale inequality

$$
\alpha_{n+1}(Q) \geq E_{Q}\left[\alpha_{n}(Q) \mid \mathscr{F}_{V_{n}^{c}}\right], \quad n=1,2, \ldots
$$

with respect to the decreasing $\sigma$-fields $\left(\mathscr{F}_{V_{n}}\right)_{n=1,2 \ldots}$. Since $Q \in \mathscr{Q}(P)$, it follows that $\left(\alpha_{n}(Q)\right)_{n=1,2 \ldots}$ is a non-negative backwards supermartingale under $Q$ which is bounded in $L^{1}(Q)$. It is thus convergent, $Q$-a.s. and in $L^{1}(Q)$, to a finite limit $\alpha_{\infty}(Q)$ such that

$$
E_{Q}\left[\alpha_{\infty}(Q)\right]=\lim _{n \rightarrow \infty} E_{Q}\left[\alpha_{n}(Q)\right]<\infty .
$$

This implies (25) and also the $P$-almost sure convergence in (24), since $Q=P$ on $\mathscr{F}_{\infty}$.

Combining Lemma 3 with the supermartingale inequality (20), we obtain the first part of the following Proposition. The second part will follow by applying the results in [14] on the behavior of consistent conditional risk measures along decreasing $\sigma$ fields.

Proposition 2. We have

$$
\rho_{\infty}(\cdot, X)=\lim _{n \rightarrow \infty} \rho_{n}(\cdot, X) \quad P-\text { a.s. }
$$

for any $X \in M$, and the kernel $\rho_{\infty}$ defines a conditional convex risk measure

$$
\rho_{\infty}: L^{\infty}(\Omega, \mathscr{F}, P) \rightarrow L^{\infty}\left(\Omega, \mathscr{F}_{\infty}, P\right)
$$

under $P$ with respect to the tail-field $\mathscr{F}_{\infty}$. This conditional risk measure has the Fatou property, and its dual representation is given by

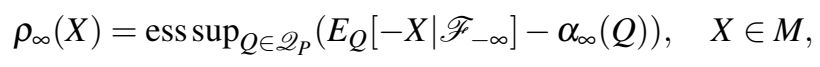

where $\alpha_{\infty}(Q)$ is given by (24). Moreover, $\alpha_{\infty}$ coincides with the minimal penalty function of $\rho_{\infty}$, i.e.,

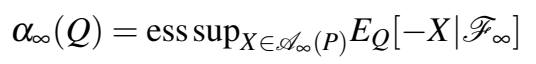

for any $Q \in \mathscr{Q}(P)$, where

$$
\mathscr{A}_{\infty}(P):=\left\{X \in L^{\infty}(\Omega, \mathscr{F}, P) \mid \rho_{\infty}(X) \leq 0 P \text { - a.s. }\right\} .
$$

Proof. 1) Take any $X \in M$ and consider the process 


$$
S_{n}(P, X)=\rho_{n}(X)+\alpha_{n}(P), \quad n=1,2, \ldots
$$

This process is bounded from below by $-\|X\|$, and the consistency condition $\rho_{n+1}=\rho_{n+1}\left(-\rho_{n}\right)$ implies the backward supermartingale inequality

$$
\left.S_{n+1}(P, X) \geq E_{P}\left[S_{n}(P, X)\right) \mid \mathscr{F}_{V_{n}^{c}}\right]
$$

see Proposition 1 for $Q=P$.

2) Take any $X \in \mathscr{A}_{n}(P)$. Since $\rho_{n}(\cdot, X) \leq 0 P$-a.s., we have

$$
\rho_{n}(\cdot, X) \leq 0 \quad \pi_{n}(\omega, \cdot)-\text { a.s. }
$$

for $P$-almost all $\omega$. Using (16) and our assumption (18), this implies

$$
E_{P}\left[-X \mid \mathscr{F}_{n}^{c}\right](\omega)=\int(-X)(\eta) \pi_{n}(\omega, d \eta) \leq c
$$

for $P$-almost all $\omega$. In view of (11), this yields the estimate

$$
\alpha_{n}(P) \leq c \quad P-\text { a.s. }
$$

This bound is valid for any $n$, and so we have $P \in \mathscr{Q}(P)$.

3) Since $P \in \mathscr{Q}(P)$, the process $\left(S_{n}(P, X)\right)_{n=1,2, \ldots}$ is a backwards supermartingale with respect to $P$ and bounded in $L^{1}(P)$, hence convergent $P$-a.s. to some finite limit $S_{\infty}(P, X)$. Combined with Lemma 3 , this yields $P$-almost sure convergence of the sequence

$$
\rho_{n}(X)=S_{n}(P, X)+\alpha_{n}(P), \quad n=1,2, \ldots
$$

to $\rho_{\infty}(X)$ and the equality

$$
\rho_{\infty}(X)=S_{\infty}(P, X)+\alpha_{\infty}(P) \quad P-\text { a.s.. }
$$

4) Since the backwards supermartingale $\left(\alpha_{n}(P)\right)_{n=1,2, \ldots}$ is bounded in $L^{1}(P)$, we can now apply the results of [14] on the limiting behavior of consistent conditional risk measures along decreasing $\sigma$-fields under a fixed reference measure $P$. Lemma 2 in [14] shows that $\rho_{\infty}$ has the Fatou property under $P$, and Theorem 4 in [14] yields the dual representation (27) and the identification of $\alpha_{\infty}$ as the minimal penalty function of $\rho_{\infty}$.

\section{Dynkin boundary and boundary risk}

In this section we are going to modify the risk kernel $\rho_{\infty}$ in such a way, that the resulting kernel $\hat{\rho}_{\infty}$ has good properties in terms of the class $\mathscr{P}$ of Gibbs measures. To this end, we use a method developed by E.B. Dynkin [8] for the construction of the entrance boundary of a Markov process, as it was applied in [10] to the integral 
representation of the class $\mathscr{P}$. This involves an extension of the local specification $\left(\pi_{V}\right)_{V \in \mathscr{V}}$ to a conditional probability distribution $\pi_{\infty}$ with respect to the tail field $\mathscr{F}_{\infty}$ which is common to all probability measures $P \in \mathscr{P}$. The following Proposition summarizes the results of $[8,9]$ and $[10]$ which are relevant for our purpose.

Proposition 3. There exists a stochastic kernel $\pi_{\infty}$ from $(\Omega, \mathscr{F} \infty)$ to $(\Omega, \mathscr{F})$ with the following properties:

i) For any $\omega \in \Omega$, the random field $\pi_{\infty}(\omega, \cdot)$ belongs to $\mathscr{P}$ and is actually an extreme point of the convex set $\mathscr{P}$. In particular we have

$$
\pi_{\infty} \pi_{V}=\pi_{\infty} \quad \text { for any } V \in \mathscr{V} .
$$

ii) For any $\omega \in \Omega$, the probability measure $\pi_{\infty}(\omega, \cdot)$ is ergodic on the tail field, that is, $\pi_{\infty}(\omega, A) \in\{0,1\}$ for any $A \in \mathscr{F}_{\infty}$, and this implies

$$
\pi_{\infty}(\eta, \cdot)=\pi_{\infty}(\omega, \cdot) \quad \pi_{\infty}(\omega, \cdot)-\text { a.s.. }
$$

iii) The kernel $\pi_{\infty}$ serves, simultaneously for all $P \in \mathscr{P}$, as a conditional distribution with respect to the tail field $\mathscr{F}_{\infty}$, that is,

$$
E_{P}\left[f \mid \mathscr{F}_{\infty}\right](\omega)=\int f(\eta) \pi_{\infty}(\omega, d \eta)
$$

$P$-a.s. for any $P \in \mathscr{P}$ and for any measurable function $f \geq 0$ on $(\Omega, \mathscr{F})$.

We endow the set $\mathscr{P}$ with the canonical $\sigma$-field $\mathscr{B}$ generated by the maps $P \rightarrow P[A] \quad(A \in \mathscr{F})$. Then the kernel $\pi_{\infty}$ can be viewed as a measurable map from $\left(\Omega, \mathscr{F}_{\infty}\right)$ to $(\mathscr{P}, \mathscr{B})$. We denote by

$$
\hat{\mathscr{F}}:=\sigma\left(\pi_{\infty}\right) \subseteq \mathscr{F}_{\infty}
$$

the $\sigma$-field on $\Omega$ generated by this map, and by

$$
\hat{M}:=M_{b}(\Omega, \hat{\mathscr{F}}) \subseteq M_{\infty}
$$

the corresponding space of bounded measurable functions. We will call $(\Omega, \hat{\mathscr{F}})$ the Dynkin boundary of the local specification $\left(\pi_{V}\right)_{V \in \mathscr{V}}$, and $\hat{\mathscr{F}}$ will be called the boundary field. Thus, any random field $P \in \mathscr{P}$ admits a representation by a probability measure on the Dynkin boundary, namely

$$
P=\hat{P} \pi_{\infty}:=\int \pi_{\infty}(\omega, \cdot) \hat{P}(d \omega),
$$

where $\hat{P}$ denotes the restriction of $P$ to the $\sigma$-field $\hat{\mathscr{F}}$. Conversely, any probability measure $\hat{P}$ on $(\Omega, \hat{\mathscr{F}})$ defines via (32) a random field $P \in \mathscr{P}$, due to (29). In this way, we obtain an integral representation of the convex set $\mathscr{P}$ that is coupled to the tail field by the kernel $\pi_{\infty}$ :

$$
\mathscr{P}=\left\{\hat{P} \pi_{\infty} \mid \hat{P} \text { is a probability measure on }(\Omega, \hat{F})\right\} .
$$


In particular, a phase transition $|\mathscr{P}|>1$ occurs if and only if the Dynkin boundary is non-trivial in the sense that the kernel $\pi_{\infty}$ really depends on the tail field, that is, not all measures $\pi_{\infty}(\omega, \cdot)$ coincide, and so $\hat{\mathscr{F}}$ does not reduce to the trivial $\sigma$-field $\{\emptyset, \Omega\}$.

Remark 2. The integral representation (32) shows that the set of extreme points of the convex set $\mathscr{P}$ is given by

$$
\mathscr{P}_{e}:=\left\{\pi_{\infty}(\omega, \cdot) \mid \omega \in \Omega\right\} .
$$

In particular, $\mathscr{P}_{e}$ is a measurable subset of $\mathscr{P}$. Denoting by $\mu_{P}$ the image of $P$ under the map $\pi_{\infty}: \Omega \rightarrow \mathscr{P}_{e}$, the representation (32) takes the form

$$
P=\int_{\mathscr{P}_{e}} Q \mu_{P}(d Q)
$$

Conversely, any probability measure $\mu$ on $\mathscr{P}_{e}$ defines via (34) a random field $P \in$ $\mathscr{P}$, and we have $\mu=\mu_{P}$. Thus we obtain a Choquet type integral representation of the convex set $\mathscr{P}$, that is, any $P \in \mathscr{P}$ is barycenter of a unique probability measure $\mu_{P}$ on the set $\mathscr{P}_{e}$ of extreme points; see $[8,9,10]$.

Let us now regularize the kernel $\rho_{\infty}$ by introducing the risk kernel $\hat{\rho}_{\infty}=\pi_{\infty} \rho_{\infty}$ defined by

$$
\hat{\rho}_{\infty}(\omega, X)=\int \rho_{\infty}(\eta, X) \pi_{\infty}(\omega, d \eta)
$$

for $\omega \in \Omega$ and $X \in M$. In order to describe its properties, we first take a closer look at the functions in the space $\hat{M}$.

Lemma 4. For any function $X \in M_{\infty}$ and any $\omega \in \Omega$, we have

$$
X(\cdot)=\hat{X}(\omega) \quad \pi_{\infty}(\omega, \cdot)-\text { a.s. }
$$

where $\hat{X}$ denotes the function in $\hat{M}$ defined by

$$
\hat{X}(\omega):=\int X(\eta) \pi_{\infty}(\omega, d \eta) .
$$

Moreover, $X$ belongs to $\hat{M}$ if and only if $X$ coincides with $\hat{X}$.

Proof. Since $\pi_{\infty}(\omega, \cdot)$ is $0-1$ on the tail field $\mathscr{F}_{\infty}$, the function $X \in M_{\infty}$ is constant $\pi_{\infty}(\omega, \cdot)$ - a.s., and this implies (36). The function $\hat{X}$ defined by (37) clearly belongs to $\hat{M}$, and so the identity $X=\hat{X}$ yields $X \in \hat{M}$. Conversely, assume that $X \in \hat{M}$, that is, $X$ is $\hat{\mathscr{F}}$-measurable. Since $\hat{\mathscr{F}}$ is generated by the map $\pi_{\infty}: \Omega \rightarrow \mathscr{P}$, there is a measurable function $f$ on $\mathscr{P}$ such that $\hat{X}(\omega)=f\left(\pi_{\infty}(\omega, \cdot)\right)$ for all $\omega \in \Omega$. This implies, for any $\omega \in \Omega$,

$$
\hat{X}(\omega)=\int f\left(\pi_{\infty}(\eta, \cdot)\right) \pi(\omega, d \eta)=f\left(\pi_{\infty}(\omega, \cdot)\right)=X(\omega),
$$


since $\pi_{\infty}(\eta, \cdot)=\pi_{\infty}(\omega, \cdot)$ for $\pi(\omega, \cdot)$-almost all $\eta$, due to (30).

Proposition 4. $\hat{\rho}_{\infty}$ is a regular convex risk kernel from $(\Omega, \hat{\mathscr{F}})$ to $(\Omega, \mathscr{F})$, and it satisfies the consistency condition

$$
\hat{\rho}_{\infty}\left(-\rho_{V}\right)=\hat{\rho}_{\infty}
$$

for any $V \in \mathscr{V}$. For fixed $\omega \in \Omega$, we have

$$
\hat{\rho}_{\infty}(\omega, \cdot) \ll \pi_{\infty}(\omega, \cdot),
$$

and the convex risk measure $\hat{\rho}_{\infty}(\omega, \cdot)$ has the Fatou property with respect to the probability measure $\pi_{\infty}(\omega, \cdot)$.

Proof. For any $X \in M$, the function $\hat{\rho}_{\infty}(\cdot, X)$ is clearly $\hat{\mathscr{F}}$-measurable. For fixed $\omega \in \Omega$, the functional $\hat{\rho}_{\infty}(\omega, \cdot)$ on $M$ inherits from $\rho_{\infty}$ the properties of a convex risk measure and also the consistency condition:

$$
\begin{aligned}
\hat{\rho}_{\infty}\left(\omega,-\rho_{V}(X)\right) & =\int \rho_{\infty}\left(\eta,-\rho_{V}(X)\right) \pi_{\infty}(\omega, d \eta) \\
& =\int \rho_{\infty}(\eta, X) \pi_{\infty}(\omega, d \eta) \\
& =\hat{\rho}_{\infty}(\omega, X) .
\end{aligned}
$$

Thus, $\hat{\rho}_{\infty}$ is a convex risk kernel from $(\Omega, \hat{\mathscr{F}})$ to $(\Omega, \mathscr{F})$ such that $\hat{\rho}_{\infty}(\omega, \cdot) \in \mathscr{R}$ for any $\omega \in \Omega$. To check its regularity, take $\hat{X} \in \hat{M}, X \in M$, and any bounded measurable function $f$ on $R^{2}$. Since $\rho_{\infty}$ is regular by Lemma 2 , and since $\hat{X}(\eta)=\hat{X}(\omega)$ for $\pi_{\infty}(\omega, \cdot)$-almost all $\eta$ by (36), we obtain

$$
\begin{aligned}
\hat{\rho}_{\infty}(\omega, f(\hat{X}, X)) & =\int \rho_{\infty}(\eta, f(\hat{X}, X)) \pi_{\infty}(\omega, d \eta) \\
& =\int \rho_{\infty}(\eta, f(\hat{X}(\eta), X)) \pi_{\infty}(\omega, d \eta) \\
& =\int \rho_{\infty}(\eta, f(\hat{X}(\omega), X)) \pi_{\infty}(\omega, d \eta) \\
& =\hat{\rho}_{\infty}(\omega, f(\hat{X}(\omega), X)) .
\end{aligned}
$$

It remains to verify the Fatou property of $\hat{\rho}_{\infty}(\omega, \cdot)$ with respect to the measure $P:=\pi_{\infty}(\omega, \cdot)$. Take any uniformly bounded sequence $\left(X_{k}\right)_{k=1,2, \ldots}$ in $M$ such that $X_{k}$ converges $P$-a.s. to some $X \in M$. Since $P \in \mathscr{P}$, Proposition 2 implies

$$
\rho_{\infty}(\cdot, X) \leq \liminf _{k \rightarrow \infty} \rho_{\infty}\left(\cdot, X_{k}\right) \quad P-\text { a.s.. }
$$

Applying Fatou's lemma, we obtain

$$
\hat{\rho}_{\infty}(\omega, X)=E_{P}\left[\rho_{\infty}(\cdot, X)\right]
$$




$$
\begin{aligned}
& \leq E_{P}\left[\liminf _{k \rightarrow \infty} \rho_{\infty}\left(\cdot, X_{k}\right)\right] \\
& \leq \liminf _{k \rightarrow \infty} E_{P}\left[\rho_{\infty}\left(\cdot, X_{k}\right)\right] \\
& =\liminf _{k \rightarrow \infty} \hat{\rho}_{\infty}\left(\omega, X_{k}\right) .
\end{aligned}
$$

In the special case $X_{k} \equiv Y$ we see that $\hat{\rho}_{\infty}(\omega, X) \leq \hat{\rho}_{\infty}(\omega, Y)$ whenever $X \leq Y$ $\pi_{\infty}(\omega, \cdot)$-a.s., and this implies $\hat{\rho}_{\infty}(\omega, \cdot) \ll \pi_{\infty}(\omega, \cdot)$.

Definition 8. Let us say that a monetary risk measure $\rho$ on $M$ has the Lebesgue property with respect to the class $\mathscr{P}$ if $\lim _{k \rightarrow \infty} \rho\left(X_{k}\right)=\rho(X)$ whenever $\left(X_{k}\right)_{k=1,2, \ldots}$ is a uniformly bounded sequence in $M$ such that

$$
\lim _{k \rightarrow \infty} X_{k}=X \quad \mathscr{P} \text { - almost surely, }
$$

that is, the convergence takes place $P$-a.s. for any $P \in \mathscr{P}$. We denote by $\mathscr{R}_{L}$ the class of all risk measures $\rho \in \mathscr{R}$ which have the Lebesgue property with respect to $\mathscr{P}$.

Remark 3. For a monetary risk measure $\hat{\rho}$ on $\hat{M}$, the Lebesgue property with respect to $\mathscr{P}$ is equivalent to the Lebesgue property with respect to pointwise convergence, that is, $\lim _{k \rightarrow \infty} \hat{\rho}\left(\hat{X}_{k}\right)=\hat{\rho}(\hat{X})$ whenever $\left(\hat{X}_{k}\right)_{k=1,2, \ldots}$ is a uniformly bounded sequence in $\hat{M}$ such that $\lim _{k \rightarrow \infty} \hat{X}_{k}(\omega)=\hat{X}(\omega)$ for any $\omega \in \Omega$. Indeed, if $\lim _{n \rightarrow \infty} \hat{X}_{n}=\hat{X} \mathscr{P}$-a.s. then the sequence converges $\pi_{\infty}(\omega, \cdot)$-a.s. for each $\omega \in \Omega$, and this amounts to pointwise convergence on $\Omega$, due to Lemma 4 .

The following theorem shows that any risk measure $\rho \in \mathscr{R}_{L}$ is uniquely determined by its behavior on the Dynkin boundary, that is, by its restriction $\hat{\rho}$ to the space $\hat{M}$.

Theorem 1. Any risk measure $\rho \in \mathscr{R}_{L}$ has the form

$$
\rho=\hat{\rho}\left(-\hat{\rho}_{\infty}\right),
$$

where $\hat{\rho}$ denotes the restriction of $\rho$ to $\hat{M}$.

Proof. Take $\rho \in \mathscr{R}_{L}$ and any $X \in M$. Since $\rho \in \mathscr{R}$, we have

$$
\rho\left(-\rho_{n}(X)\right)=\rho(X)
$$

for any $n \geq 1$. The sequence $\left(\rho_{n}(X)\right)_{n=1,2, \ldots}$ is uniformly bounded by $\|X\|$, and Proposition 2 shows that

$$
\lim _{n \rightarrow \infty} \rho_{n}(\cdot, X)=\rho_{\infty}(\cdot, X) \quad \mathscr{P} \text { - almost surely. }
$$

Now note that, for any $\omega \in \Omega$, the equality

$$
\rho_{\infty}(\cdot, X)=\int \rho_{\infty}(\eta, X) \pi_{\infty}(\omega, d \eta)=\hat{\rho}_{\infty}(\omega, X)=\hat{\rho}_{\infty}(\cdot, X)
$$


holds $\pi_{\infty}(\omega, \cdot)$-almost surely, due to Lemma 4 . In view of the integral representation (32), this implies $\rho_{\infty}(\cdot, X)=\hat{\rho}_{\infty}(\cdot, X) P$-a.s. for any $P \in \mathscr{P}$, and so we get

$$
\lim _{n \rightarrow \infty} \rho_{n}(\cdot, X)=\hat{\rho}_{\infty}(\cdot, X) \quad \mathscr{P} \text { - almost surely }
$$

Applying the Lebesgue property of $\rho$ with respect to $\mathscr{P}$, we obtain

$$
\rho(X)=\lim _{n \rightarrow \infty} \rho\left(-\rho_{n}(\cdot, X)\right)=\rho\left(-\hat{\rho}_{\infty}(\cdot, X)\right)=\hat{\rho}\left(-\hat{\rho}_{\infty}(\cdot, X)\right),
$$

and this proves the representation (40).

Remark 4. If a risk measure $\rho \in \mathscr{R}$ has the Fatou property with respect to $\mathscr{P}$ but not the Lebesgue property, then the preceding proof yields the inequality $\rho \leq \hat{\rho}\left(-\hat{\rho}_{\infty}\right)$.

Now suppose that the risk kernel $\hat{\rho}_{\infty}$ is such that each risk measure $\hat{\rho}_{\infty}(\omega, \cdot)$ has not only the Fatou property but also the Lebesgue property with respect to the measure $\pi_{\infty}(\omega, \cdot)$; this condition is clearly satisfied in the entropic case of Remark 1. In such a situation we have $\mathscr{R}_{L} \neq \emptyset$, and there is a one-to-one correspondence between the class $\mathscr{R}_{L}$ and the class $\hat{\mathscr{R}}_{L}$ of all convex risk measures $\hat{\rho}$ on $\hat{M}$ that have the Lebesgue property with respect to pointwise convergence:

Corollary 1. If each risk measure $\hat{\rho}_{\infty}(\omega, \cdot)$ has the Lebesgue property with respect to the measure $\pi_{\infty}(\omega, \cdot)$, then we have

$$
\mathscr{R}_{L}=\left\{\rho\left(-\hat{\rho}_{\infty}\right) \mid \hat{\rho} \in \hat{\mathscr{R}}_{L}\right\},
$$

and in particular $\mathscr{R}_{L} \neq \emptyset$.

Proof. The inclusion " $\subseteq$ " follows from the preceding theorem. Conversely, if $\hat{\rho} \in$ $\hat{\mathscr{R}}_{L}$ then $\rho:=\hat{\rho}\left(-\hat{\rho}_{\infty}\right)$ clearly defines a convex risk measure on $M$ which belongs to the class $\mathscr{R}$. To see that $\rho$ has the Lebesgue property with respect to $\mathscr{P}$ and thus belongs to $\mathscr{R}_{L}$, take a uniformly bounded sequence $\left(X_{n}\right)$ in $M$ such that $X_{n} \rightarrow X$ $\mathscr{P}$-a.s.. In particular, the convergence holds $\pi_{\infty}(\omega, \cdot)$-a.s. for any $\omega \in \Omega$, and this implies $\lim _{n \rightarrow \infty} \hat{\rho}_{\infty}\left(\omega, X_{n}\right)=\hat{\rho}_{\infty}(\omega, X)$. Thus we have pointwise convergence of the uniformly bounded sequence $\left(\hat{\rho}_{\infty}\left(\cdot, X_{n}\right)\right)_{n=1,2, \ldots}$ in $\hat{M}$. Since $\hat{\rho}$ belongs to $\hat{\mathscr{R}}_{L}$, we get

$$
\lim _{n \rightarrow \infty} \rho\left(X_{n}\right)=\lim _{n \rightarrow \infty} \hat{\rho}\left(-\hat{\rho}_{\infty}\left(\cdot, X_{n}\right)\right)=\hat{\rho}\left(-\hat{\rho}_{\infty}(\cdot, X)\right)=\rho(X) .
$$

This proves the converse inclusion "’”. In particular we have $\mathscr{R}_{L} \neq \emptyset$, since $\hat{\mathscr{R}}_{L} \neq \emptyset$. Indeed, any probability measure $\hat{P}$ on the Dynkin boundary induces via

$$
\hat{\rho}(X)=\int(-X) d \hat{P}
$$

a convex risk measures $\hat{\rho} \in \hat{\mathscr{R}}_{L}$.

Corollary 2. A risk measure $\rho \in \mathscr{R}_{L}$ is uniquely determined by the local specification $\left(\rho_{V}\right)_{V \in \mathscr{V}}$ if and only if the local specification $\left(\pi_{V}\right)_{V \in \mathscr{V}}$ admits no phase transition, i.e., 


$$
\left|\mathscr{R}_{L}\right|=1 \Longleftrightarrow|\mathscr{P}|=1
$$

Proof. If $|\mathscr{P}|=1$ then $\hat{\mathscr{F}}$ is trivial, $\hat{M}$ can be identified with $R^{1}$, and there is only one monetary risk measure on $\hat{M}$ given by $\hat{\rho}(m)=-m$. Thus (41) implies $\left|\mathscr{R}_{L}\right|=$ 1. Conversely, if $|\mathscr{P}|>1$ then we can choose $\omega_{1}, \omega_{2} \in \Omega$ such that $\pi_{\infty}\left(\omega_{1}, \cdot\right) \neq$ $\pi_{\infty}\left(\omega_{2}, \cdot\right)$. Taking

$$
A=\left\{\omega \in \Omega \mid \pi_{\infty}\left(\omega_{1}, \cdot\right)=\pi_{\infty}\left(\omega_{2}, \cdot\right)\right\} \in \hat{\mathscr{F}},
$$

we obtain $\pi_{\infty}\left(\omega_{1}, A\right)=1$ und $\pi_{\infty}\left(\omega_{2}, A\right)=0$ due to (30). But $\hat{\rho}_{\infty}\left(\omega_{i}, \cdot\right) \ll \pi_{\infty}\left(\omega_{i}, \cdot\right)$ for $i=1,2$ by Proposition 4 , and so we get $\hat{\rho}_{\infty}\left(\omega_{1},-I_{A}\right)=1$ and $\hat{\rho}_{\infty}\left(\omega_{2},-I_{A}\right)=0$. This shows that the two risk measures $\hat{\rho}_{i}:=\hat{\rho}_{i}(\omega, \cdot) \in \mathscr{R}_{L}$ do not coincide, and so we have $\left|\mathscr{R}_{L}\right|>1$.

The absence of a phase transition at the underlying probabilistic level implies $\left|\mathscr{R}_{L}\right|=1$, but not $|\mathscr{R}|=1$, as illustrated by the following remark on the entropic case.

Remark 5. Let us return to the special case of local law invariance in Remark 1, where the local risk measures $\rho_{V}(\omega, \cdot)$ are of the entropic form (19) with some parameter $\beta_{\infty}(\omega)$ which depends on the tail field $\mathscr{F}_{\infty}$. For fixed $\omega \in \Omega$, the measure $\pi_{\infty}(\omega, \cdot)$ is ergodic on $\mathscr{F}_{\infty}$, and so we have

$$
\beta_{\infty}(\eta)=\hat{\beta}(\omega):=\int \beta_{\infty}(\zeta) \pi_{\infty}(\omega, d \zeta)
$$

for $\pi_{\infty}(\omega, \cdot)$-almost all $\eta \in \Omega$. Thus the risk kernel $\hat{\rho}_{\infty}=\pi_{\infty} \rho_{\infty}$ in (35) takes the form

$$
\hat{\rho}_{\infty}(\omega, X)=\frac{1}{\hat{\beta}(\omega)} \log \int e^{-\hat{\beta}(\omega) X(\eta)} \pi_{\infty}(\omega, d \eta)
$$

Clearly, the convex risk measure $\hat{\rho}_{\infty}(\omega, \cdot)$ has not only the Fatou property but also the Lebesgue property with respect to the probability measure $\pi_{\infty}(\omega, \cdot)$. Thus we can apply Corollary 1 and Corollory 2.

In the absence of a phase transition we have $\mathscr{P}=\{P\}$ for a single random field $P$. In this case, the $\hat{\mathscr{F}}$-measurable function $\hat{\beta}$ reduces to the constant

$$
\beta:=\int \beta_{\infty}(\omega) P(d \omega) \in[0, \infty),
$$

and the unique risk measure $\rho$ in $\mathscr{R}_{L}$ is given by the entropic risk measure (5) with respect to $P$ and $\beta$. In particular we obtain $\rho(X)=E_{P}[-X]$ for any function $X \in M_{\infty}$, since $X(\cdot)=E_{P}[X] P$-almost surely, due to the ergodicity of $P$ on $\mathscr{F}_{\infty}$. On the other hand, the convex risk measures $\rho_{\infty}(\omega, \cdot)$ in (21) all belong to $\mathscr{R}$ due to $(22)$, and they are different from $\rho$ since regularity of the kernel $\rho_{\infty}$ implies $\rho_{\infty}(\omega, X)=-X(\omega)$ for any $X \in M_{\infty}$. 


\section{Acknowledgement}

We began to look at spatial risk measures while the first author was visiting the Departments of Mathematics of LMU and TU in München. Special thanks are due to Codina Cotar who participated in these initial discussions. Further results were obtained while the first author was visiting the FIM at ETH Zürich, the Hausdorff Institute of Mathematics at the University of Bonn. and the Department of Mathematics of the National University of Singapore. The support of these institutions is gratefully acknowledged.

\section{References}

1. Adrian,T. and Brunnermeier, M. K., CoVaR. Preprint Federal Reserve and Princeton University (2011)

2. Artzner, P., Delbaen, F., Eber, J.-M., and Heath, D., Coherent measures of risk. Mathematical Finance 9, 203 - 228 (1999)

3. Acciaio, B. and Penner, I., Dynamic risk measures. In: G. Di Nunno and B. Oksendal (Eds.) Advanced Mathematical Methods for Finance, 1-34, Springer (2011)

4. Bion-Nadal, J., Dynamic risk measures: time-consistency and risk measures from BMO martingales. Finance \& Stochastics 12(2), 219-244 (2008)

5. Delbaen, F., Differentiability properties of utility functions. In: Delbaen, F., Rásonyi, M. and Stricker, C. (Eds.) Optimality and Risk - Modern Trends in Mathematical Finance, 39-48. Springer, Berlin (2009)

6. Dobrushin, R. L.: Description of a random field by means of conditional probabilities and conditions of its regularity, Theor. Probability Appl. 13, 197 - 224 (1968)

7. Detlefsen, K. and Scandolo, G., Conditional and dynamic convex risk measures, Finance \& Stochastics 9(4), 539 - 561 (2005)

8. Dynkin, E. B., Integral representation of excessive measures and excessive functions. Russ. Math. Surveys 27, 43 - 84 (1972)

9. Dynkin, E. B., Entrance and exit spaces for a Markov process. Actes Congrès. Intern. Math. 2, 507 - 512 (1971)

10. Föllmer, H., Phase Transition and Martin Boundary, Séminaire de Probabilités (Strasbourg) 9, 305 - 317 (1975)

11. Föllmer, H., Spatial risk measures and their local specification: The locally law-invariant case, Statistics \& Risk Modeling 31 (1), 79 - 101, (2014)

12. Föllmer, H. and Knispel, Th., Convex risk measures: Basic facts, law-invariance and beyond, asymptotics for large portfolios. In: L.C. MacLean and W.T. Ziemba (Eds) Handbook of the Fundamentals of Financial Decision Making, Part II, 507 - 554, World Scientific (2013)

13. Föllmer, H. and Penner, I., Convex risk measures and the dynamics of their penalty functions, Statistics \& Decisions 24 (1), 61-96, (2006)

14. Föllmer, H. and Penner, I., Consistent risk measures and a non-linear extension of backwards martingale convergence, Preprint (2014)

15. Föllmer, H. and Schied, A., Stochastic Finance-An Introduction in Discrete Time, 3rd edition, Graduate Textbook Series, Walter de Gruyter \& Co., Berlin, (2011)

16. Föllmer, H. and Schied, A., Convex measures of risk and trading constraints, Finance and Stochastics 6(4), 429 - 447 (2002)

17. Frittelli, M. and Rosazza Gianin, E., Putting order in risk measures, Journal of Banking and Finance 26, 1473 - 1486 (2002)

18. Georgii, H.-O., Gibbs Measures and Phase Transitions, 2nd ext. edition, De Gruyter Studies in Mathematics 9 (2011) 
19. Kupper, M. and Schachermayer, W., Representation results for law invariant time consistent functions, Math. Financ. Econ. 2(3), 189 - 210 (2009) 